\title{
Truths and Myths of Radiotherapy
}

\author{
Federico Lorenzo ${ }^{1 *}$, Georgina Miralda ${ }^{2}$, Sergio Aguiar ${ }^{3}$ and Aldo Quarneti ${ }^{4}$ \\ ${ }^{1}$ Assistant Professor, Radiation Oncology Department, Udelar \\ ${ }^{2}$ Resident, Radiation Oncology Department, Udelar \\ ${ }^{3}$ Associate Professor, Radiation Oncology Department, Udelar \\ ${ }^{4}$ Chief Professor, Radiation Oncology Department, Udelar
}

Submission: May 13, 2019; Published: May 28, 2019

"Correspondence Author: Federico Lorenzo, Radiation Oncology Department. “Dr. Manuel Quintela” Clinics Hospital, Faculty of Medicine, University of the Republic (Udelar), Montevideo, Uruguay, South America

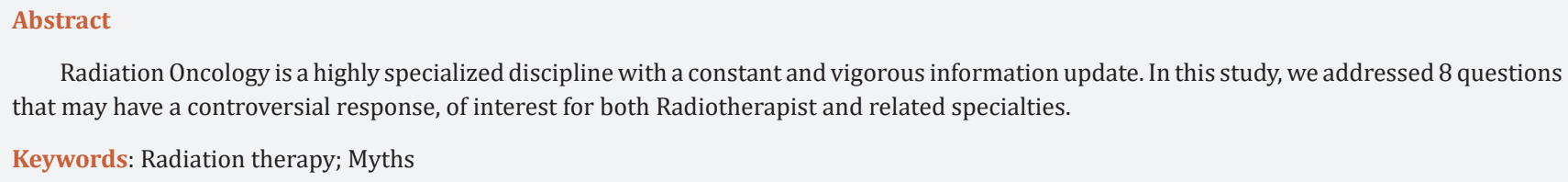

Radiation Oncology is a highly specialized discipline with a constant and vigorous information update. In this study, we addressed 8 questions that may have a controversial response, of interest for both Radiotherapist and related specialties.

Keywords: Radiation therapy; Myths

\section{Introduction}

Radiation oncology is a highly specialized therapeutic discipline that requires important radiophysical, radiobiological and clinical knowledge. Radiotherapy uses ionizing radiation, mainly X-rays, gamma and accelerated electrons, and its relationship with living matter as therapeutic means. It is a procedure of physical nature, of locoregional action fundamentally. Working day to day in this exciting discipline, we have heard some concepts that can be controversial, in this study we carry out a systematized bibliographic search to answer these questions.

i. It is contraindicated to wash the irradiated skin during Radiotherapy for breast cancer.

ii. A previously irradiated site of head and neck can't be re-irradiated.

iii. Irradiation at the breast CTV by tangent fields achieves an acceptable coverage of axillary nodal levels I and II.

iv. The "triple plan" treatment and the use of antifungals is effective for the prophylaxis of mucositis in head and neck radiotherapy.

v. Radiotherapy of verrucous carcinoma (Akerman) is contraindicated.

vi. Radiotherapy is a risk factor for the development of second tumors. vii. Renal carcinoma is "radioresistant".

viii. The effectiveness of radiotherapy decreases with a hemoglobin lower than $10 \mathrm{~g} / \mathrm{dl}$.

\section{Materials and Methods}

A bibliographic search was performed in pubmed using the key words referring to the proposed controversial statements, sorting searches by reference articles, recent studies and clinical trials. All the abstracts of the selected papers were read in a first instance and at last, a second review was made to choose a maximum of five articles per statement.

\section{It Is Contraindicated to Wash the Irradiated Skin During Radiotherapy for Breast Cancer}

Radiation therapy techniques for breast cancer have evolved over time. These can affect the incidence and severity of skin reactions. Acute radiation reactions occur between the second and fourth week of treatment and may persist for two to four weeks after the end of treatment. Washing the treated skin could play a preventive role by reducing the incidence of wet desquamation, caused by bacterial and fungal growth, which increase the inflammatory response and damage to basal cells [1].

In a randomized study, Campbell and Illingworth [2] analyzed two hypotheses for washing deprivation: first, moistening 
the treated skin increased the severity of the acute skin reaction. Second, they state that it is not the water itself, but the use associated with soap and friction caused by washing and drying, which damages the skin. The patients who performed skin wash presented less pruritus than those who did not wash, with a statistically significant difference. Another associated sign was erythema, which was significantly reduced in patients who performed skin washing. In the wash group, desquamation decreased by the eighth week of treatment, while those of non-washing continued to rise. Little difference was found between the washing groups with water alone and with soap and water; overall, acute skin reactions were lower in patients who wash the treated skin.

In a study by Roy et al. [3], 33\% of the patients who did not wash their skin presented wet desquamation, compared to $14 \%$ of those who did. Although the results were not statistically significant, they showed a tendency to lower toxicity. This may indicate that there is actually a positive effect, but that it has not been demonstrated due to the limited number of patients. Studies have demonstrated that skin washing influences the acceptance of treatment, and we believe that it should not be discouraged during radiotherapy treatment in breast cancer.

\section{A Previously Irradiated Site of Head and Neck Can't Be Re-Irradiated}

Until the 1970s, there were no studies about irradiating previously treated sites of head and neck, since the concept of "non-re-irradiable" tissues was the premise of those times due to the concern of possible serious side effects. By the early 1990s, the first publications with a significant number of patients began. One of the main challenges in any re-irradiation plan is the correct selection of patients, with re-staging studies including PET-CT, and thus achieve a balance between disease control and adverse effects. In this selection, it is relevant to consider some factors such as patient's performance status, GTV, radiation dose received previously, time elapsed between the first course of radiotherapy and the new one [4] De Crevoisier [5] re-irradiated 169 patients with head and neck carcinoma at radical doses, obtaining an average survival of 10 months and a rate of side effects grade 3 or 4 of $36 \%$ and grade 5 of $3 \%$.

More recent studies analyze different types of fractionation, use of brachytherapy, fractionated stereotactic radiotherapy plans, obtaining 2-year local control rates of $30-40 \%$. There have been further studies with new technologies, such as IMRT, and using different and to pursuit better conformational doses. Lee, $\mathrm{N}$ [6] re irradiated 105 patients with IMRT technique, with an average dose of 59.4 Gy, obtaining a global survival rate at 2 years of $37 \%$ and a rate of severe late complications of $11 \%$. Salama et al. [7], re irradiate 115 patients with a significant difference in overall survival at 3 years of $3 \%$ vs $30 \%$ in a dose cohort of 58 Gy Therefore, it is plausible to irradiate head and neck tumors, considering a multidisciplinary behavior and individual characteristics of the patients.

\section{Irradiation at The Breast CTV By Tangent Fields Achieves an Acceptable Coverage of Axillary Nodal Levels I and II}

Several factors such as patient's age, lymph node status, molecular profile, among others, influence in the decision of treating axillary territories in an adjuvant treatment for breast cancer. In some occasions, it is argued to contour only level III, inferring that levels I and II are covered satisfactorily by tangent fields. In our review we found several studies that address this issue, with a heterogeneous coverage of such levels. Belkacemi et al. [8], analyzed the coverage by classic tangent fields with 3D conformal radiotherapy in 105 patients, obtaining in level I a dose range from 1 to $57 \mathrm{~Gy}$, and in level II from 0 to $46 \mathrm{~Gy}$; $95 \%$ of the prescribed dose was received by of $10 \%$ of level I and $3 \%$ of level II, increasing this coverage in the $50 \%$ isodose curve to $45 \%$ and $11 \%$ respectively.

Reznick et al. [9] analyzed a series of 35 patients. The results obtained suggest that tangential fields designed to treat only the breast, do not cover adequately the axillary region, therefore, prophylactic therapy of the axilla cannot be relied upon. Thus, obtaining a better conformational dose in level II over level I. Another study included 15 patients with tumours no greater than $25 \mathrm{~mm}$, who underwent conservative surgery and adjuvant radiotherapy. The average dose at level I was $48.7 \%$ with a range of 5 to $80 \%$ [10]. According to the analyzed studies, the dose coverage at the axillary levels I and II is heterogeneous, insufficient and therefore not acceptable for adjuvant treatment at such levels.

\section{The "Triple Plan" Treatment and the Use of Antifungals Is Effective for The Prophylaxis of Mucositis in Head and Neck Radiotherapy}

Oral mucositis occurs in approximately $20 \%$ up to $40 \%$ of patients receiving conventional chemotherapy, and almost all patients who receive radiation therapy. Typically, it appears at the second or third week after starting the treatment and may persist for months [11]. Mucositis can affect the continuity of the radiotherapy, due to severe pain, which also affects nutritional intake, oral hygiene and quality of life. It has been demonstrated that doses as low as 1000-2000 cGy can cause mucositis. Benzydamine hydrochloride is a topical nonsteroidal agent that has anti-inflammatory, analgesic/anesthetic, and antimicrobial effects. There have been various studies that demonstrate a reduction in the severity of mucositis in patients with head and neck cancer. Benzydamine mouthwash is well tolerated, and favorable effects continue even in the seventh week of treatment when the majority of patients had received the total dose of more than 60 Gy [12].

The mucosal barrier injury related to radiation allows microbial colonization and infection, which in turn leads to the amplification of tissue injury. The prevention of a candida infection should reduce the severity of radiation mucositis [13]. 
The "triple plan" treatment is usually used for the prophylaxis of mucositis secondary to radiotherapy. This consists of combining a topical anesthetic (lidocaine), an antihistamine (diphenhydramine) and an antacid (aluminum or magnesium hydroxide); accompanied or not by an antifungal agent. Miller et al. [14], in a phase III, randomized study, evaluated the efficacy of mouthwashes with oral doxepin and triple therapy with diphenhydramine/lidocaine/antacid versus placebo in the treatment of oral mucositis. Pain related to radiotherapy was reduced significantly, compared to placebo.

The most frequently isolated species from patients during radiotherapy are Candida albicans and Candida glabrata. Given the high prevalence of oral fungal infections during the treatment, mycological examinations are recommended to identify their species and determine their sensitivity to drugs in order to prevent complications. Another study evaluated prophylaxis with fluconazole and its impact on the severity of oral mucositis during head and neck radiotherapy. There was a significantly beneficial impact on the incidence of prolonged interruptions of radiotherapy related to mucositis, grade 3 or 4, and supported the hypothesis that candidiasis plays an important role in the severity of radiation mucositis [15].

According to the MASCC / ISSO guidelines [11], an evidencebased recommendation is the use of benzydamine mouthwash or low-level laser therapy to prevent oral mucositis in patients with head and neck cancer, receiving a moderate dose radiation therapy without concomitant chemotherapy. There was no evidence regarding the use of sucralfate in the prevention or treatment of mucositis in patients receiving chemo-radiotherapy. The triple plan showed benefits in pain relief, however there is no evidence regarding the prevention of mucositis. On the other hand, there is evidence that the use of fluconazole and benzydamine can prevent this effect.

\section{Radiotherapy of Verrucous Carcinoma (Akerman) Is Contraindicated}

Verrucous carcinoma is an unusual variant of well differentiated squamous cell carcinoma, which was first described in the oral cavity by Lauren Ackerman in 1947. He noted that this tumor had a distinctive morphological appearance and a specific clinical behavior, therefore it should be separated from the other squamous cell carcinomas. Verrucous carcinoma has a favorable prognosis even with locoregionally advanced lesions [16]. Three arguments have been exposed in detriment of radiotherapy: anaplastic transformation, increased incidence of post-radiotherapy metastasis, and less local control with radiotherapy vs surgery.

Given the peculiarity and histopathological difficulties in the diagnosis of this pathology, in our research, we found less than 5 retrospective studies and no prospective clinical trials. These studies report from 0 to $7 \%$ of anaplastic transformation after radiant treatment, also occurring in patients undergoing surgical treatment. No increase in the incidence of post-radiotherapy metastasis was demonstrated in any of the series reported [17-19]. Although local control was significantly greater with surgery than radiotherapy $85 \%$ vs $47.7 \%$, surgically rescued patients have the same local control rate compared with patients operated on the first instance [17]. Therefore, we can emphasize that patients suitable for surgical resection with low morbidity, is a good primary choice. Patients who require surgery with high impact on morbidity, radiotherapy is a possible option, and in no case "anaplastic transformation" will affect the therapeutic approach.

\section{Radiotherapy Is A Risk Factor for The Development of Second Tumors}

Carcinogenesis is one of the most challenging stochastic effects of radiotherapy. The main risk factors measured in the studies of second tumors have been age, size of the treatment field, the radiotherapy technique, and the use of chemotherapy, among others. It is estimated that cancer survivors have a $14 \%$ higher risk of developing a second tumor compared to the general population. Tumors are considered secondary when they have the following characteristics: they are caused by ionizing radiation effects, they appear inside the radiation field, those histologically different from the primary, they have a latency period of several years, they do not exist at the time of initial diagnosis, and that the patient is not a carrier of a cancerprone syndrome [20].

A systematic review of clinical and epidemiological studies measured the dose-volume distribution and second reported tumors. These happens in approximately 3 to $40 \%$ of cancer survivors, in a period of 10 to 40 years after the diagnosis of the first tumor. According to the data obtained, they suggest that the risk of second tumors in organs and tissues adjacent to or near the target volume, increases with larger fields of irradiation [21]. In a study with patients who had second tumors, treated previously with radiotherapy, $50 \%$ of them were in the range of the initial treatment volume, and $10 \%$ within the field. There was an increase in the frequency of tumors within the volume of low doses of less than 6 Gy. The latency period for the clinical manifestation of the second tumors was 18 years for low energy $\mathrm{X}$-rays and 6 years for gamma rays [22].

The choice of the least toxic radiation modality in children and adolescents is essential due to their high radio sensitivity and lower body surface area. A study of pediatric patients with brain, head and neck tumors, compared the advantages of proton therapy with respect to the IMRT and VMAT techniques. They suggest that the advantage of proton therapy is its ability to limit the exposure of neighboring organs due to the lack of exit dose. Therefore, the risk of a second malignant disease is lower when protons are used compared to conventional photon therapy or IMRT techniques [23]. The evidence shows that radiotherapy is a risk factor for the development of second tumors. Modern 
techniques, including IMRT, SBRT and particle therapies, can deliver highly conformed dose distributions, allowing dose escalation to the target volume, while decreasing it to adjacent normal tissues, thus reducing the subsequent risk of second tumors.

\section{Renal Carcinoma Is "Radioresistant"}

The incidence of renal cell carcinoma is $3 \%$ of the tumors in adults. A large percentage of them are diagnosed in locoregionally advanced stages, given their retroperitoneal location, and usually for not presenting symptoms until the tumor has a considerable volume. The lymph node involvement, renal capsule extension, involvement of the renal vein and inferior venous thrombosis, are well known as indicators of poor prognosis, especially if patients present two or more of these factors [24]

In these patients, therapeutic strategies have been sought after surgical treatment in order to improve overall and/or disease-free survival. For that matter, the role of radiotherapy has been discussed, taking into account the concept of "radio resistance". Three prospective studies were carried out between the $70 \mathrm{~s}$ and the $80 \mathrm{~s}$, in which the results have been unclear, unfortunately these works used old techniques, extensive treatment fields and high fractionation [25-27]. In recent years, several retrospective studies with diverse results were presented. Tunio et al. [28] performed a meta-analysis in which they concluded that post-operative radiotherapy reduces locoregional failure significantly, without an impact on the rate of disease-free survival or global survival. Up to date, there is no clear evidence of the benefit of adjuvant radiotherapy in renal carcinoma. New randomized clinical trials with new techniques and modern technologies are required to unveil this question.

\section{The Effectiveness of Radiotherapy Decreases with A Hemoglobin Lower Than 10 G/Dl}

Over the years, several studies have shown an association between the hemoglobin level and the effectiveness of radiotherapy. The effects of ionizing radiation on tumor cells are based on the formation of oxygen free radicals that react with DNA, inducing cell death. It is presumed that hemoglobin supplies oxygen to tumor cells, therefore, hypoxia is a determining factor for radio resistance. A radiation dose, approximately twice or three times higher, is needed to destroy hypoxic cells compared to well oxygenated cells. A low concentration of hemoglobin is a predictor for a decrease in local control and survival after radiotherapy for cancer of the cervix, bladder, lung, and head and neck [29].

In a retrospective analysis, the effects of the hemoglobin level with respect to the tumor response were compared in patients with cervical cancer, taking values between $10 \mathrm{~g} /$ $\mathrm{dl}$ and $>11 \mathrm{~g} / \mathrm{dl}$. In the accelerated hyper fractionation group, patients with $\mathrm{Hb}>11 \mathrm{~g} / \mathrm{dl}$ had a greater complete response, statistically significant, compared with those with $\mathrm{Hb}$ of 10 $10.9 \mathrm{~g} / \mathrm{dl}$. There was no significant difference in the group that received concurrent chemotherapy and radiotherapy. The relative benefit of an adequate level of hemoglobin appears to be much greater for patients treated with exclusive radiotherapy than for patients treated with concurrent chemoradiotherapy. It is possible that the radio sensibilization effect of the improved hemoglobin levels may compensate, to some extent, the non-use of concurrent chemotherapy [30].

Another study evaluated the effect of low hemoglobin levels pre-treatment in locally advanced squamous cell carcinoma of the head and neck, with complete resection. Hemoglobin levels of $<12$ and $>12 \mathrm{~g} / \mathrm{dl}$ were associated with 3-year survival rates of $55 \%$ and $87 \%$, respectively, and 5 -year survival rates of $25 \%$ and $71 \%$, respectively $(\mathrm{p}<0.001)$. Therefore, they suggest that tumor cell oxygenation and the correction of anemia appear to be important even after the $\mathrm{R} 0$ resection [31].

Lee WR et al. [32]. confirm the importance of the hemoglobin level in a prospective, randomized trial in patients with advanced squamous cell carcinoma of the head and neck. Hemoglobin levels were stratified as normal $(>14.5 \mathrm{~g} / \mathrm{dl}$ for men,> $13 \mathrm{~g}$ / dl for women) or anemic ( $<14.5 \mathrm{~g} / \mathrm{dl}$ for men, $<13 \mathrm{~g} / \mathrm{dl}$ for women). Anemic patients according to conventional criteria, before starting radiotherapy had a reduction of approximately $40 \%$ in survival and increased $30 \%$ the locoregional failure compared to similar patients with normal hemoglobin levels. There is evidence that tumor control is improved with optimal hemoglobin levels, however, no consensus has been reached regarding the exact minimum level that should be maintained during radiotherapy treatment.

\section{References}

1. McQuestion M (2011) Evidence-based skin care management in radiation therapy: clinical update. Semin Oncol Nurs 27(2): 1-17.

2. Campbell IR, Illingworth MH (1992) Can patients wash during radiotherapy to the breast or chest wal? A randomized control trial. Clin. Oncol (R Coll Radiol) 4(2): 78-82.

3. Roy I, Fortin A, Larochelle M (2001) The impact of skin washing with water and soap during breast irradiation: a randomized study. Radiother Oncol 58(3): 333-339.

4. Chen AM, Phillips TL, and Lee NY (2011) Practical considerations in the re-irradiation of recurrent and second primary head-and-neck cancer. Int J Radiat Oncol Biol Phys 81(5): 1211-1219.

5. De Crevoisier R, Bourhis J, Domenge C (1998) Full dose reirradiation for unresectable head and neck carcinoma: experience at the GustaveRoussy Institute in a series of 169 patients. J Clin Oncol 16 (11):35563562.

6. Lee N, Chan K, Bekelman JE (2007) Salvage re-irradiation for recurrent head and neck cancer. Int J Radiat Oncol Biol Phys 68(3): 731-740.

7. Salama JK, Vokes EE, Chmura (2006) Long-term outcome of concurrent chemotherapy and reirradiation for recurrent and second primary head-and-neck squamous cell carcinoma. Int J Radiat Oncol Biol Phys 64 (2): 382-391.

8. Belkacemi Y, Allab-Pan Q Bigorie V (2013) The standard tangential fields used for breast irradiation do not allow optimal coverage and dose distribution in axillary levels I-II and the sentinel node area. Ann Oncol 24(8): 2023-2028. 


\section{Cancer Therapy \& Oncology International Journal}

9. Reznik J, Cicchetti MG, Degaspe B (2005) Analysis of axillary coverage during tangential radiation therapy to the breast. Int J Radiat Oncol Biol Phys 61(1):163-168.

10. Orecchia R, Huscher A, Leonardi MC (2005) Irradiation with standard tangential breast fields in patients treated with conservative surgery and sentinel node biopsy: using a three-dimensional tool to evaluate the first level coverage of the axillary nodes. Br J Radiol 78: 51-54.

11. Lalla RV, Bowen J, Barasch A (2014) MASCC/ISOO clinical practice guidelines for the management of mucositis secondary to cancer therapy. Cancer 120(10):1453-1461.

12. Sheibani KM, Mafi AR, Moghaddam S (2015) Efficacy of benzydamine oral rinse in prevention and management of radiation-induced ora mucositis: a double-blind placebo controlled randomized clinical trial. Asia Pac J Clin Oncol 11(1): 22-27.

13. Nicolatou-Galitis O, Velegraki A, Sotiropoulou-Lontou A (2006) Effect of fluconazole antifungal prophylaxis on oral mucositis in head and neck cancer patients receiving radiotherapy. Support Care Cancer 14(1): 44-51.

14. Miller RC, Le-Rademacher J, Sio TTW (2016) A phase III, randomized double-blind study of doxepin rinse versus Magic Mouthwash versus placebo in the treatment of acute oral mucositis pain in patients receiving head and neck radiotherapy with or without chemotherapy (Alliance A221304). Int J Radiat Oncol Biol Phys 96(5): 938.

15. Kurnatowski P, Moqbil S, Kaczmarczyk D (2014) Signs, symptoms and the prevalence of fungi detected from the oral cavity and pharynx of radiotherapy subjects with head and neck tumors, and their susceptibility to chemotherapeutics. Ann Parasitol 60(3): 207-213.

16. Ackerman L (1948) Verrucous Carcinoma of the Oral Cavity. Surgery 23:670-678.

17. Tharp ME, Shidnia H (1995) Radiotherapy in the treatment of verrucous carcinoma of the head and neck. The Laryngoscope.; 105(4): 391-396.

18. Ferlito A, Rinaldo A, Mannara GM (1998) Is primary radiotherapy an appropriate option for the treatment of verrucous carcinoma of the head and neck? J Laryngol Otol 112(2): 132-139.

19. Huang SH, Lockwood G, Irish J, Ringash J, Cummings B, et al. (2009) Truths and myths about radiotherapy for verrucous carcinoma of larynx. Int J Radiat Oncol Biol Phys 73(4): 1110-1115.

20. Travis L, Bhatia S, Allan J (2011) Second primary cancers. In: DeVita VJ, Lawrence T, Rosenberg S, (eds.,) Cancer: Principles and Practice of Oncology. 9th ed. Philadelphia, PA: Lippincott Williams and Wilkins: 2393.
21. Journy N, Mansouri I, Rodriguez A (2018) Volume effects of radiotherapy on the risk of second primary cancers: A systematic review of clinical and epidemiological studies. Radiotherapy and Oncology 131: 150-159.

22. Dörr W, Thomas Herrmann (2002) Second Primary Tumors after Radiotherapy for Malignancies: Treatment-Related Parameters. Strahlenther Onkol 178: 357-362.

23. Moteabbed M, Yock T, Paganetti H (2014) The risk of radiation-induced second cancers in the high to medium dose region: a comparison between passive and scanned proton therapy, IMRT and VMAT for pediatric patients with brain tumors. Phys. Med. Biol. 59: 2883-2899.

24. Sene P, Hunt R, McMahon T (1992) Renal Carcinoma in Patients Undergoing Nephrectomy: Analysis of Survival and Prognostic Factors. British Journal of Urology 70(2): 125-134.

25. Finney R (1973) The value of radiotherapy in the treatment of hypernephroma: a clinical trial. Br J Urol 45: 258-269.

26. Kjaer M, Frederiksen P, Engelholm S (1987) Postoperative Radiotherapy in Stage II and III Renal Adenocarcinoma. A Randomized Trial by the Copenhagen Renal Cancer Study Group. International Journal of Radiation Oncology \& Biology \& Physics 13(5): 665-672.

27. Kjaer M, Iversen P, Hvidt V (1987) A randomized trial of postoperative radiotherapy versus observation in stage II and III renal adenocarcinoma. A study by Copenhagen Renal Cell Cancer Study Group. Scand J Urol Nephrol 21(4): 285-289.

28. Tunio M A, Hashmi A, Rafi M (2010) Need for a new trial to evaluate postoperative radiotherapy in renal cell carcinoma: a meta-analysis of randomized controlled trials. Ann Oncol 21(9):1839-1845.

29. Becker A, Stadler P, Lavey RS (2000) Severe anemia is associated with poor tumor oxygenation in head and neck squamous cell carcinomas. Int J Radiat Oncol Biol Phys 46(2): 459-466.

30. Thakur P, Seam RK, Gupta MK (2015) Comparison of effects of hemoglobin levels upon tumor response among cervical carcinoma patients undergoing accelerated hyperfractionated radiotherapy versus cisplatin chemoradiotherapy. Asian Pac J Cancer Prev 16(10): 4285-4289.

31. Rades D, Seidl D, Janssen S (2016) The effect of low hemoglobin levels on outcomes of radiotherapy following microscopically complete resection of locally advanced SCCHN: Implications for the future. Journal of Cranio-Maxillo-Facial Surgery 44(9): 1441-1444.

32. Lee WR, Berkey B, Marcial V (1998) Anemia is associated with decreased survival and increased locoregional failure in patients with locally advanced head and neck carcinoma: A secondary analysis of RTOG 85 - 27. Int J Radiat Oncol Biol Phys 42(5): 1069 - 1075.

\section{Your next submission with Juniper Publishers will reach you the below assets}

- Quality Editorial service

- Swift Peer Review

- Reprints availability

- E-prints Service

- Manuscript Podcast for convenient understanding

- Global attainment for your research

- Manuscript accessibility in different formats

( Pdf, E-pub, Full Text, Audio)

- Unceasing customer service

Track the below URL for one-step submission https://juniperpublishers.com/online-submission.php 